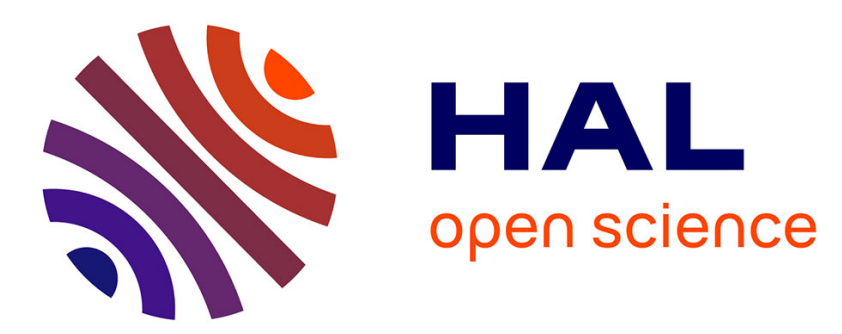

\title{
Dislocation clustering and long-range internal stresses in monotonically and cyclically deformed metal crystals
}

\author{
H. Mughrabi
}

\section{To cite this version:}

H. Mughrabi. Dislocation clustering and long-range internal stresses in monotonically and cyclically deformed metal crystals. Revue de Physique Appliquée, 1988, 23 (4), pp.367-379. 10.1051/rphysap:01988002304036700 . jpa-00245783

\section{HAL Id: jpa-00245783 https://hal.science/jpa-00245783}

Submitted on 1 Jan 1988

HAL is a multi-disciplinary open access archive for the deposit and dissemination of scientific research documents, whether they are published or not. The documents may come from teaching and research institutions in France or abroad, or from public or private research centers.
L'archive ouverte pluridisciplinaire HAL, est destinée au dépôt et à la diffusion de documents scientifiques de niveau recherche, publiés ou non, émanant des établissements d'enseignement et de recherche français ou étrangers, des laboratoires publics ou privés. 
Classification

Physics Abstracts

$46.30 \mathrm{~J}-81.40 \mathrm{~L}-62.20 \mathrm{~F}$

\title{
Dislocation clustering and long-range internal stresses in monotonically and cyclically deformed metal crystals
}

\author{
H. Mughrabi
}

Institut für Werkstoffwissenschaften, Lehrstuhl I, Universität Erlangen-Nürnberg, Martenstrasse 5, 8520 Erlangen, F.R.G.

(Reçu le 26 mai 1987, accepté le 14 août 1987)

RESUME. - On passe en revue les structures de dislocations en parois et cellules dans les cristaux métalliques déformés, en insistant sur l'hétérogénéité de distribution des dislocations. L'évolution de la sous-structure de dislocations est décrite en terme d'écrouissage et restauration dynamique. L'article insiste sur le modèle dit "composite" dans lequel la distribution hétérogène des dislocations est considérée comme un matériau composite constitué de parties dures et molles correspondant aux parois et intérieurs des cellules, respectivement. Des contraintes internes à longue portée, d'amplitude cohérente avec l'expérience, font partie du modèle composite. Elles sont une conséquence des conditions de compatibilité en cours de déformation. Le modèle composite permet une nouvelle compréhension de la contrainte macroscopique d'écoulement plastique et une bonne description du chargement inverse, y compris l'effet Bauschinger dans le cas des monocristaux.

\begin{abstract}
Dislocation wall and cell structures in deformed metal crystals are reviewed briefly, emphasizing the heterogeneity of the dislocation distribution. The evolution of the dislocation substructure is discussed in terms of work hardening and dynamic recovery. The main part of the paper deals with the so-called composite model in which the heterogeneous dislocation distribution is considered as a composite consisting of bonded hard and soft components corresponding to cell walls and cell interiors, respectively. Long-range internal stresses whose magnitude is consistent with experiment are an integral part of the composite model. They arise as a necessary consequence of the compatibility requirements during deformation. The composite model leads to a new understanding of the macroscopic flow stress and to a good description of reverse loading including the Bauschinger effect in the case of single crystals.
\end{abstract}

\section{INTRODUCTION}

The dislocation microstructure of deformed metals is characterized by the mutual elastic interaction of the dislocations and by their tendency to cluster in order to form dislocation tangles, walls and, in most cases, three-dimensional dislocation cell structures, for recent reviews compare $[1,2]$. Whereas dislocation tangles (braids, bundles) and walls can form under single-slip conditions, the formation of three-dimensional dislocation cell structures is typical of combined single and secondary slip or multiple slip. The heterogeneity of the dislocation distribution is an important feature common not only to monotonically but al so to cyclically strained metals [3].

Dislocation cell structures form both at low and at high temperatures, differing, however, in their qualitative appearance, as illustrated in Fig. 1. At low temperatures the cell walls are "untidy" and have non-negligible thicknesses (Fig. 1a). At higher temperatures they become much sharper and approach ideal low-angle boundary configurations (Fig. 1b).
The cell structures encountered in fatigue can be considered to be of an intermediate type in the sense that, for example, $\alpha$-iron $[6,7]$ and copper [8] fatigued moderately at room temperature exhibit cell structures of the low-temperature type which approach cell structures of the high-temperature type after extensive cyclic deformation. Frequently, misorientations from cell to cell are found. It appears, though this is not documented systematical$1 y$ in the literature, that the misorientations across cell walls of the low-temperature type are much smaller $[4,9]$ than in the case of the planar subcell boundaries typical of high-temperature deformation. Qualitatively, this can be related to the fact that while the "thicker" cell walls formed at low temperatures contain dislocations of both signs and hence have a dipolar character, the low-angleboundary-type cell walls characteristic of high temperatures consist predominantly of dislocations of only one sign. At a given temperature, the misorientations increase with increasing deformation [4-8]. 
The present contribution will focus primarily on the athermal deformation behaviour at low temperatures at which diffusion plays a marginal role. Hence, thermally activated dislocation glide will not be considered explicitly. Following a brief, formal description of work hardening and dynamic recovery, the emphasis will be placed on recently developed so-called composite (dislocation) models of the flow stress in which deformation-induced long-range internal stresses are intimately related to the heterogeneity of the dislocation distribution. The sources of these long-range internal stresses will be reviewed. Attention will be drawn to the competing effects of energy reduction by dislocation clustering and energy enhancement by the extra energy due to the deformation-induced long-range internal stresses in clustered dislocation arrays. Finally, it will be shown that the composite model lends itself readily to a description of reversed loading including the Bauschinger effect and that the shape of the cyclic hysteresis loop of single crystals is governed by the composite nature of plastic deformation.

a)

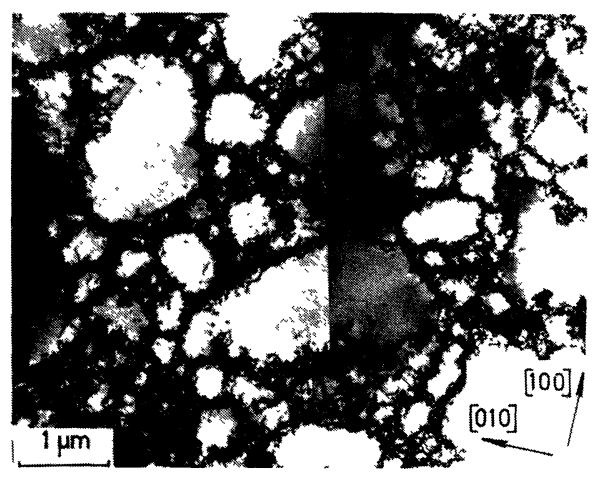

b)

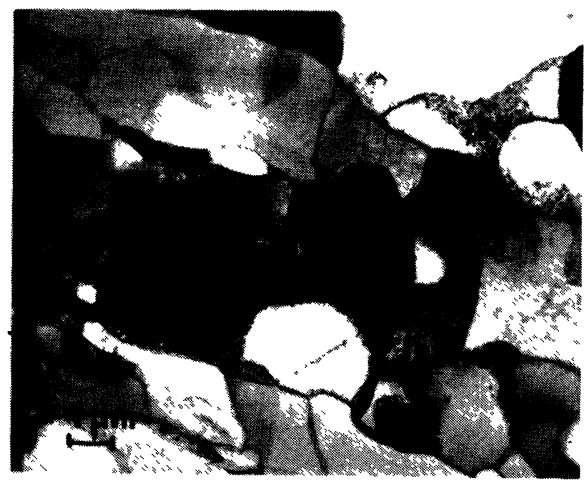

Fig. 1. Dislocation cell structures.

a) [001]-orientated copper single crystal, deformed in tension at $293 \mathrm{~K}$ [4].

b) Aluminium, deformed in tensile creep at $473 \mathrm{~K}$

[5]. Courtesy of W. B? um and G. König.

\section{KINETICS OF DISLOCATION ACCUMULATION, MORK HARDENING, DYMAMIC RECOVERY AND STEADY-STATE DEFORMATION.}

Following ref. [10], the differential increase of the dislocation density $P_{i}$ of dislocations of type $i$, e.g. edge or screw, which contribute a fraction $c_{j}$ to the total plastic shear strain $r$ is given by

$$
d \rho_{i}=\frac{2}{b L_{i}} d \gamma-2 \rho_{i} y_{i} c_{i} \frac{d \gamma}{b} .
$$

Here $L_{i}$ is the slip path of dislocations of type $i$, $b$ the modulus of the Burgers vector and $y_{j}$ the socalled annihilation length. The latter implies that when two dislocations of opposite sign encounter each other on slip planes separated by a distance equal to or smaller than $y_{j}$, then mutual annnihilation will occur. Thus the first term in eq. (1) represents the differential increase of the dislocation density due to the multiplication of the dislocations and the second term takes into account the differential strain-induced decrease of the dislocation density. Hence, the second term describes the dynamic recovery of dislocations.

Considering only edge ( $i=e)$ and screw ( $i=s)$ segments, $c_{s} \approx c_{e} \approx 0.5$. If all screw dislocations annihilate mutually by stress-induced cross slip between unlike screw dislocations, then eq. (1) with $i=e$ would describe the kinetics of the accumulation of the whole density of dislocations of one slip system. If the density of screw dislocations is nonnegligible, the whole dislocation density is given by that of the non-screw dislocations multiplied by a factor of, at most, two. In the following this factor will be ignored so that eq. (1) describes the behaviour for one slip system. If $n$ slip systems operate in multiple slip, the total dislocation density $\rho$ will be given by

$$
\rho=n \cdot \rho_{\mathbf{j}},
$$

and the fraction $c_{\mathbf{j}}$ will be given approximately by

$$
c_{i} \approx 0.5 / n \text {. }
$$

In order to describe the stress-strain behaviour we assume that the shear flow stress $\tau$ is related to the total dislocation density $\rho$ by the generally accepted relation [11]

$$
\tau=\alpha \mathrm{Gb} \sqrt{\rho},
$$

where $\alpha$ is a geometrical constant. In the case of the Hirsch-Saada process, $\alpha \approx 0.4[12,13]$.

Differentiation of eq. (4) yields

$$
\frac{d \tau}{d \rho}=\frac{\alpha G b}{2 \sqrt{\rho}}=\frac{(\alpha G b)^{2}}{2 \tau} .
$$

By inserting eqs. (4) and (5) into eq. (1) and by substituting $\rho$ for $n \cdot p_{i}$ via eq. (2) we obtain

$$
\frac{b L_{i}{ }^{\tau}}{n(\alpha G b)^{2}-n c_{i} y_{i} L_{i} \tau^{2}} d \tau=d \gamma .
$$

With the assumption that $n=4$ slip systems operate and that $c_{i}$ is given by eq. (3) we can write (1eaving away the index i)

$$
\frac{2 b L \tau}{2 n(\alpha G b)^{2}-y L \tau^{2}} d \tau=d \gamma \text {. }
$$

Eqs. (6a) or (6b) can be integrated. For this purpose, however, certain assumptions must be made with regard to the dependence of the slip path $L$ of the 
dislocations and of the annihilation length $y$ on the flow stress $\tau$ (or on the shear strain $\gamma$ ). Before proceeding further, it must al so be noted that eqs. (6a) and (6b) do not take the heterogeneity of the dislocation distribution into account at all. Strictly speaking, they apply only to homogeneous (uniform) dislocaton distributions. They might thus be applied locally in regions of relatively uniform dislocation distribution such as, in the simplest case, the cell wall or the cell interior regions. In this case, the $s 1$ ip path $L$ would have to be related to the lateral dimensions of these regions in the glide plane, namely the cell wall thickness $d_{w}$ or the diameter $d_{c}$ of the cell interior regions, respectively.

Bearing these restrictions in mind, we now return to the question of integrating eqs. (6a) and (6b). Regarding the dislocation slip path $L$, two cases are considered, namely $L=$ const. and $L=K_{2} / \tau$. The first case would apply, for example, to stage I work hardening of face-centred cubic (fcc) single crystals and the second case to stage II work hardening [10]. For the annihilation distance we assume $y=$ const. or $y=k_{1} / T$. The first relation has been found to apply quite well (with $y_{e} \approx 1.6 \mathrm{~nm}$ ) for pure edge dislocation arrays in copper crystals [10], the second implies a mutual dislocation trapping mechanism leading to spontaneous annihilation, for example by some form of cross slip [14]. The parameters $K_{1}$ and $K_{2}$ are considered to be constants at a given temperature and strain rate. In lack of a detailed theory they should be evaluated from experimental data on local dislocation densities in the case of $K_{1}$ and, in the case of $k_{2}$ from the characteristic dimensions of the dislocation distribution and from slip line data. Very plausible results have been obtained for arbitrary dislocations in cell structures in deformed copper crystals with $K_{1}=2.14 \mathrm{~N} / \mathrm{m}$ [14].

The combination of these possibilities yields four cases $(a, b, c, d)$ for which eq. (6a) has been integrated. The results are summarized in Table 1. For $L=$ const. a saturation of the flow stress (with a saturation stress $\tau_{s}$ and saturation dislocation density $\left.\rho_{s}\right)$ is obtained in cases a) $(y=$ const.) and b) $\left(y=K_{1} / \tau\right)$. For $L=K_{2} / \tau$, saturation occurs for $y=$ const. in case $c$ ), whereas for $y=k_{1} / \tau$ in case d) linear hardening is obtained.

It should be noted that the annihilation distance $y$ is expected to depend on temperature $T$, strain rate 8 and stacking fault energy SFE [15]. The exact dependence should follow from a theory yielding $y=y(\dot{\gamma}, T, S F E)$. Qualitatively, it is expected that decreasing $\dot{\gamma}$, increasing $T$ and increasing SFE will cause y to increase. The consequences would be a reduction of $\tau_{s}$ and $\rho_{s}$ in cases $a, b)$ and c) in which saturation occurs and a decrease of the linear work-hardening rate in case d).

In order to describe the deformation of a wall or a cell structure, the coupled deformation of the walls of high dislocation density and the dislocation-poor regions between the walls must be considered as in ref. [10]. In more detailed models of the evolution of the dislocation substructure Prinz and Argon [16] and Nix, Gibeling and Hughes [17] consider dynamic recovery to occur mainly between the walls, whereas in the walls static recovery is assumed to be dominant. We note here, that, in a formal manner, we can al so describe static recovery in our approach by a term similar to the second term in eq. (1), using an effective value of the annihilation distance $y$ that is appropriate at a particular temperature and strain rate.

\section{THE COMPOSITE MODEL}

\subsection{Deformation by single slip.}

The composite model represents an attempt to describe in a simplistic fashion the deformation behaviour of a crystal in which the dislocations are distributed heterogeneously in the form of a wall or a cell structure. In the following, an outline will be given of the composite model in the form in which it was first proposed on the basis of microstructural TEM (transmission electron microscopy) studies on fatigued copper single crystals orientated for single slip $[1,18,19]$ and 1 ater al so for tensile-deformed crystals oriented for multiple slip $[1,4]$. A very similar model was developed by Pedersen and co-workers in order to describe the Bauschinger effect [20] and the cyclic deformation [21] of copper single crystals of single-slip orientation. With regard to the details, some differences exist between these two related models and also with respect to other models $[22,23]$ which, in the present context, will only be noted briefly in passing.

Fig. 2 shows the typical dislocation distribution observed by TEM in copper single crystals of singleslip orientation deformed cyclically into saturation (at room temperature). In a (12人1) section (Fig. 2a) which contains the primary Burgers vector $b_{p}=\frac{1}{2}[\overline{101}]$ and lies perpendicular to the primary glide plane (111), persistent slip bands (PSBs) with the so-called ladder or wall structure, embedded in the vein or bundle structure of the matrix $(M)$, are observed. The walls and the veins consist essentially of a very high density of elongated primary edge dislocation dipole loops and are separated by "channels" of rather low dislocation density $[3,10,18,24-26]$. Since plastic deformation is concentrated strongly in the PSBS which carry a plastic shear strain amplitude of almost $1 \%$ as compared to only about $10^{-4}$ in the case of the matrix, we shall confine our considerations to plastic flow in the wall structure of the PSBs. The details of the dislocation distribution in the wall structure are shown in Fig. 2b. Dislocation glide in the channels occurs by the bowing-out of edge segments from the walls across the channels and by the spreading of the screw dislocations along the channels, as illustrated schematically in Fig. 3a [18]. A necessary consequence of the steady-state conditions prevailing in cyclic saturation is that a dynamic equilibrium must exist between the processes of dislocation multiplication and annihilation with regard to both screw and edge dislocations [10].

Aside from the gross features of the dislocation distribution described above, the following more subtle observation is important. In Fig. $2 b$ which shows the dislocation distribution pinned in the stress-applied state, it is evident that dislocation segments near the walls are much more strongly curved than those in the centre of the channels. In particular, attention is drawn to the large number of strongly curved short edge segments bowing out at the periphery of the walls. The implications are that the locally acting shear stress varies strongly 
across the channels, increasing sharply near the walls $[1,18,19,24,26]$. The results of a quantitative evaluation of the variation of the local shear stresses from wall to wall $[1,19]$ are shown in Fig. $4\left(x / / d_{c}\right.$ is the distance from the "left" to the "right" wall, normalized with respect to the channel width $d_{f}$ ). Considering the external saturation shear flow stress $\tau_{s}=28 \mathrm{MPa}$ as a macroscopic flow stress $\hat{\boldsymbol{\tau}}$, it is to be noted that the local shear stress $\tau_{\text {loc }}$ is significantly lower than $\boldsymbol{\tau}^{2}$ in the centre of the channels and much higher near the walls. Mechanical equilibrium requires that the local shear stresses in the walls be approximately $100 \mathrm{MPa}[1,19\}$.

It is concluded that significant long-range internal stresses exist in the wall structure in the stress-applied state, as shown schematically in Fig. 3b. The origin of such long-range internal stresses which were first noted in 1973 [27] is not immediately clear, since the bulk of the dislocations are edge-dislocation di- or multipoles with no significant long-range internal stress fields. It will be shown below that the composite model explains the experimental observation very satisfactorily.

The basic idea underlying the composite model is that a crystal containing a heterogeneous dislocation distribution behaves mechanically like a twophase (or multi-phase) material even if it is chemi- cally single-phase. Referring to Fig. 2b and ignoring details of the dislocation distribution for the moment, the view is taken that the material is a composite consisting of bonded dark and light components of high and low dislocation density which have correspondingly high and low local flow stresses, respectively. When such a composite is strained, the deformations of the components must be compatible. The simplest assumption is that the components are strained in parallel under the condition that the total strain be constant throughout the structure. This assumption is based on the consideration that deformation occurs by dislocation glide and that the glide planes are continuous through the soft and the hard components. Other simplifying assumptions are that both components exhibit ideal elastic-plastic behaviour and that they possess the same elastic constants.

The composite model, as described above, is formally similar to Masing's model of the yielding of polycrystals [28]. Masing considered the polycrystal as an aggregate of bonded crystallites of varying orientations with respect to the stress axis and with accordingly different yield stresses. He described the overall yielding behaviour under forward and reverse straining by assuming that the grains are strained in parallel under the condition of constant total (axial) strain and that they behave in an ideal elastic-plastic manner. It follows that our composite model of a single crystal is formally

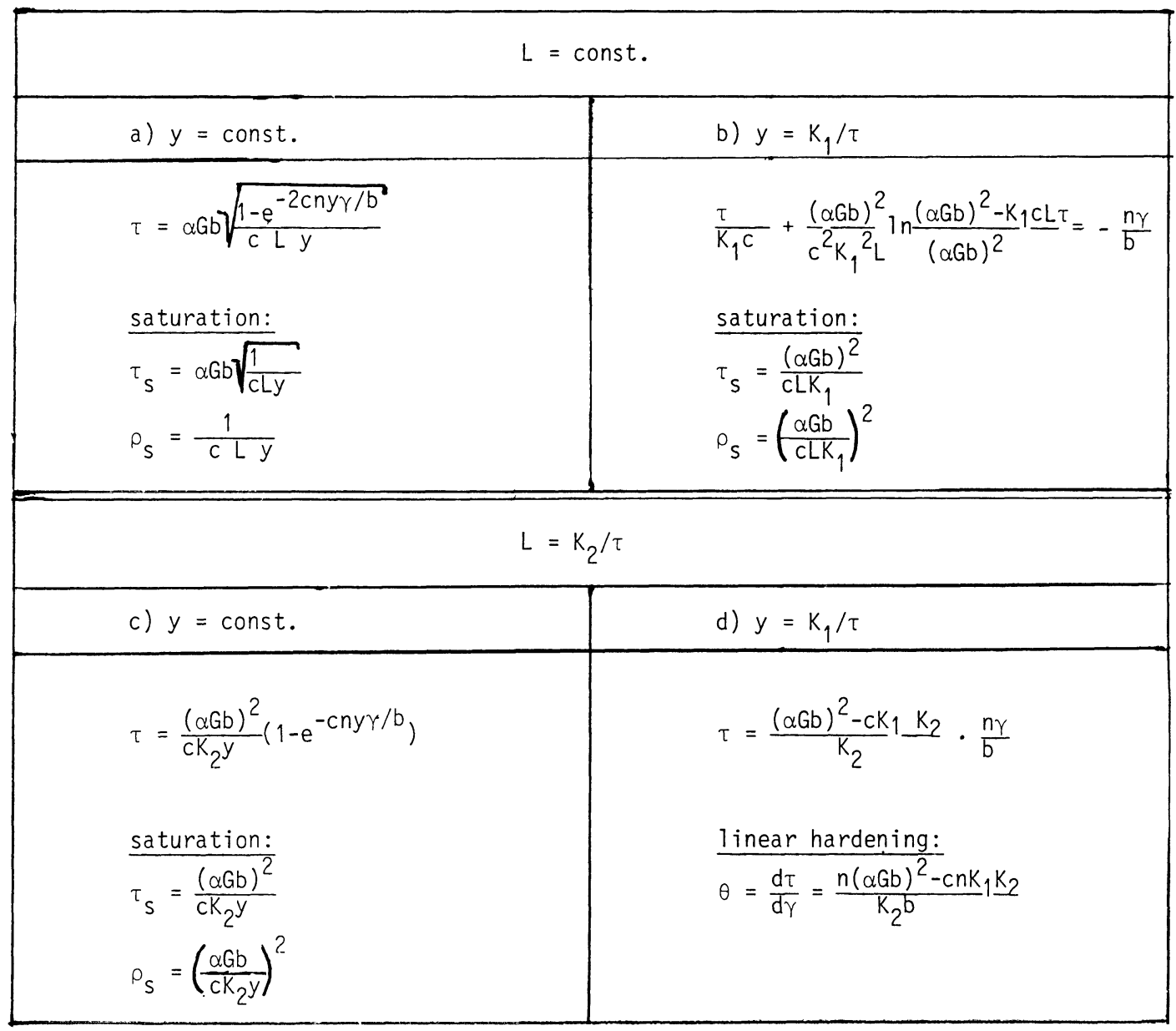

Table 1: Solutions of eq. (6a) for different combinations of slip path $L$ and annihilation distance $y$. 
equivalent to a two-component Masing model.

The "hypothetical" stress-strain curves of the components 1 and 2 and of the composite are shown in Figs. $5 \mathrm{a}$ and $5 \mathrm{~b}$, respectively. For an elucidation of the term "hypothetical" the reader is referred to ref. [1]. The macroscopic behaviour of the composite results from the superposition of the microscopic behaviours of the components under due consideration of the area fractions $f_{1}$ and $f_{2}$ occupied by components 1 and 2 in the glide $p l$ ane, respectively. For all cases to be considered in the following, these area fractions are identical with the volume fractions. Yielding of the composite can be divided conveniently into microyielding corresponding to plastic yielding of the soft component while the hard component is still deforming elastically and into macroyielding when the material has become fully plastic. An important feature of the composite model is that long-range internal stresses are built-in as an integral part. Upon unloading, when the applied stress is reduced to zero, these long-range internal stresses are frozen in (residual stresses) in the form of forward (tensile) stresses $\Delta \sigma_{1}$ and backward (compressive) stresses $\Delta \sigma_{2}$ in the hard and in the soft components, respectively.

The two-component Masing model can be formulated for the wall structure of the PSBs (Fig. 2) in simple relations $[1,18,19]$ in terms of the shear stresses $\tau$ and the shear strains $r$ as follows. The subscripts $w$ and $c$ refer to walls (component 1 ) and channels (component 2 ), respectively. The compatibility requirement is

a)

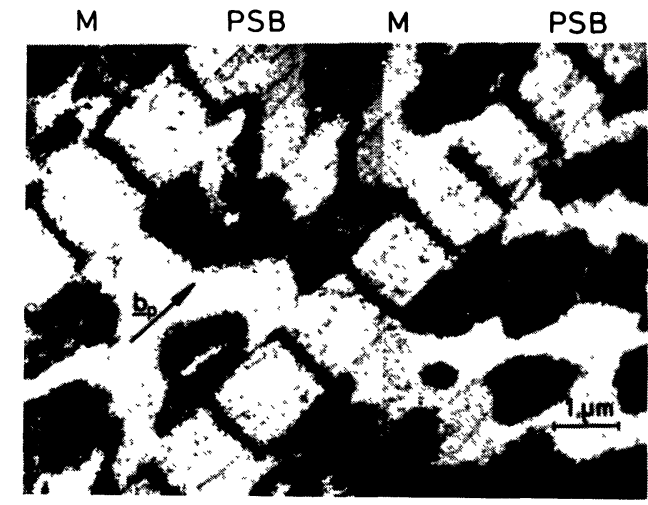

b)

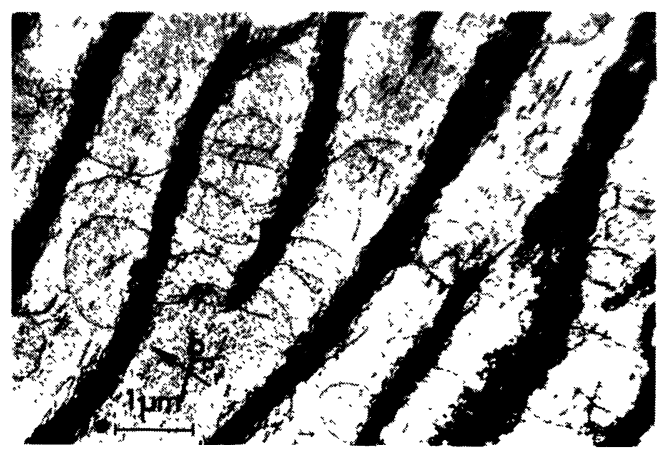

Fig. 2. Dislocation arrangement in fatigued copper single crystals [24].

a) (121)-section showing matrix (M) and persistent slip bands (PSB).

b) Section parallel to primary glide plane (111) in PSB wall structure.

$$
\gamma_{t}=\gamma_{e l}+\gamma_{p l}=\text { const., }
$$

where the subscripts $t$, el and $p l$ refer to total, elastic and plastic, respectively. The applied stress for a given value of $\gamma_{t}$ is given by a rule of mixtures:

$$
\begin{aligned}
& \tau=f_{c} \tau_{c}\left(\gamma_{t}\right)+f_{w} \tau_{w}\left(\gamma_{t}\right), \\
& \text { with } f_{c}+f_{w}=1 .
\end{aligned}
$$

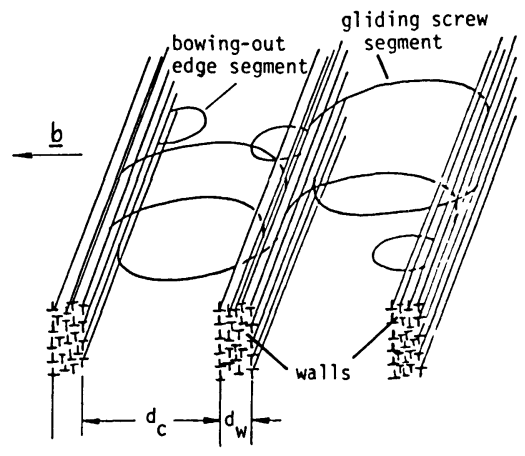

a)
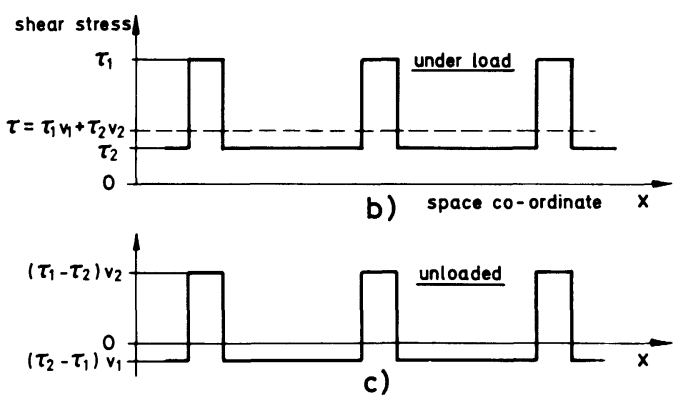

Fig. 3. Schematic illustration of dislocation behaviour in the PSB wall structure. After [18].

a) Gilide of edge and screw dislocations.

b) Idealized local stress distribution in the stress-applied state.

c) Idealized local stress distribution in the unloaded state. ( $v_{1}, v_{2}$ : volume fractions)

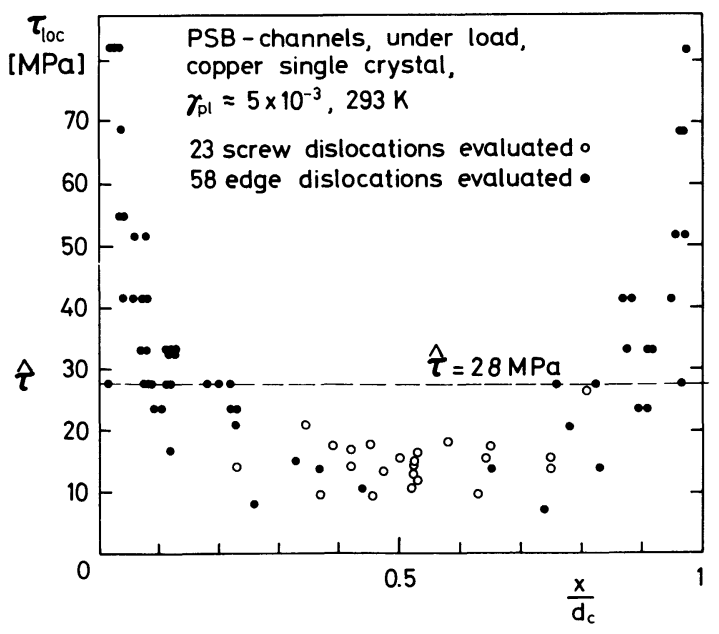

Fig. 4. Variation of the local stress in the PSB channels in the stress-applied state at a plastic shear strain amplitude $\gamma_{\mathrm{pl}}=5 \cdot 10^{-3}$ [19]. 


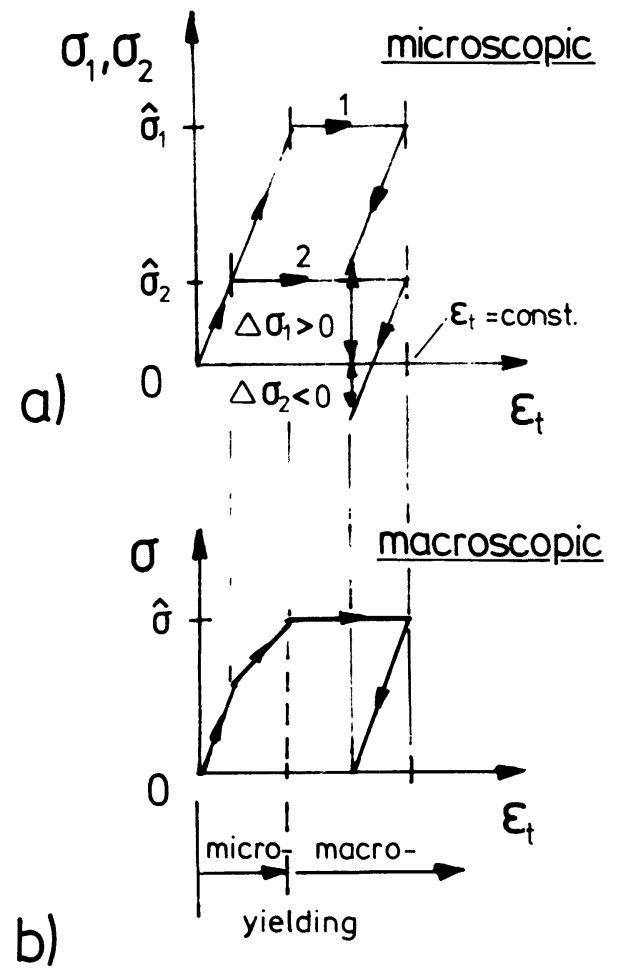

Fig. 5. Hypothetical stress-strain curves for a twocomponent composite model during forward straining and unloading. After $[1,19]$.

a) Microscopic behaviour of the components.

b) Macroscopic behaviour of the composite.

$\left(\sigma_{1}, \sigma_{2}\right.$ and $\sigma$ are the current (axiai) stresses of components 1 and 2 and of the composite, respectively, and $\hat{\sigma}_{1}, \hat{\sigma}_{2}$ and $\hat{\sigma}$ are the corresponding (axial) flow stresses, $\varepsilon_{t}$ is the total (axial) strain.)

At the point of macroyielding eq. (8a) reads

$$
\hat{\tau}=f_{c} \hat{\tau}_{c}+f_{w} \hat{\tau}_{w},
$$

where $\hat{\tau}_{\text {and }} \hat{\tau}_{w}$ denote the local flow stresses and $\tilde{\tau}$ the overall macroscopic flow stress. The local flow stresses $\hat{\tau}_{c}$ and $\hat{\tau}_{w}$ are related to the macroscopic flow stress $\hat{\tau}$ as follows

$$
\begin{aligned}
\hat{\tau}_{C} & =\hat{\tau}+\Delta \tau_{c} \\
\text { with } \Delta_{\tau_{c}} & =-\Gamma G f_{W}\left(\gamma_{p l, c}-\gamma_{p l, w}\right) \\
\text { and } \hat{\tau}_{W} & =\hat{\tau}+\Delta \tau_{w} \\
\text { with } \Delta \tau_{w} & =+\Gamma G f_{c}\left(\gamma_{p l, c}-\gamma_{p l, w}\right),
\end{aligned}
$$

where $\Gamma$ is Eshelby's elastic accommodation factor [29] to which we shall return later. The stresses $\Delta \tau_{c}$ and $\Delta T_{W}$ are deformation-induced longrange internal stresses which arise from the plastic strain mismatch between the soft and the hard components, as expressed by eqs. (11) and (13). They enhance the applied stress in the hard components and oppose it in the soft components, ensuring overall compatibility. Upon unloading, they are fully retained if no reverse plastic flow occurs and only partially otherwise, compare $[1,18,19]$. In the unloaded state, $\tau=0$, and eq. ( $8 a)$ reduces to

$$
f_{c} \Delta \tau_{c}+f_{w} \Delta \tau_{w}=0 .
$$

Figs. $3 b$ and $3 c$ summarize schematically the local shear stress distribution in the PSB wall structure in the stress-applied and in the unloaded states. The rectangular stress profile is, of course, an idealization. More detailed considerations [1] lead to a more realistic profile which agrees closely with that of Fig. 4.

The sources of the long-range internal stresses are the edge dislocations of like sign deposited at the interfaces between walls and channels, as indicated in Fig. 3a. The density of these interface dislocations represents only a few percent of the total disTocation density [1].

The most important feature of the composite model is its ability to explain the observed long-range internal stresses in a straightforward manner. In addition, the composite model provides a better understanding of the macroscopic flow stress via eqs. (10) to (13), depending on whether the local flow stress $\hat{\tau}_{f}$ of the soft or $\hat{\tau}_{w}$ of the hard regions is considered as a reference. In the former case $\tau_{\text {f }}$ is equal to $\hat{\tau}_{c}$ plus the back stress $\left|\Delta \tau_{c}\right|$, in the latter case it is equal to $\hat{\tau}_{w}$ minus the forward stress $\left|\Delta T_{W}\right|$.

Returning to the Eshelby factor, it must be remarked that this quantity is not included in a simple Masing-type model and represents a refinement adopted from the continuum theory of dispersion hardening by Brown and co-workers $\{30\}$ and emphasized in papers by Pedersen and co-workers $[20,21]$ in the present context. The Eshelby factor $\Gamma$ depends on the shape of the "inclusions", i.e. of the walls in the present case, and on the mode of straining. It lies between zero and one. In the opinion of Pedersen et al. [21], an appropriate value for the walls would be $T \cong 1 / 100$. The consequences of such a low value would be a strong reduction of the longrange internal stresses $\Delta \tau_{c}$ and $\Delta \tau_{w}$ whereas, in the present author's opinion, the experimental results strongly indicate that $T$ must be close to 1 [19]. In fact, a value of $T=1$ will be used throughout this paper as in earlier work $[1,18,19]$.

The choice of the value $\Gamma \approx 1$ is derived from the experimental observations. The justification is briefly as follows. The TEM observations of dislocations pinned in the stress-applied state (Fig. $2 \mathrm{~b}$ ) indicate that dislocations glide into and out of the walls. In situ TEM studies [31] support this picture. Hence, it can be concluded that the hard dislocation walls are not only strained elastically but do in fact yield plastically $[1,18,19]$. The required relatively high local stresses are provided by the applied stress plus the aiding forward internal stress $\Delta \tau$, cf. eq. (12). According to the measurements of the local shear stresses (Fig. 4), the shear stress acting locally in the walls is about $100 \mathrm{MPa}$ which is considered of the right magnitude in order to cause dislocation glide in the walls. If, on the other hand, $\Gamma$ were much smaller than one, as suggested by Pedersen et al. [21], $\Delta \tau_{w}$ would be reduced significantly. In this case, neither the large values of the long-range internal stresses deduced from the observations nor the 
bowing-out of small edge segments from the walls could be explained in terms of the composite model. As will be shown later, the analysis of the shape of the hysteresis loop al so leads to the conlusion that the walls yield plastically (and that $\Gamma=1$ ).

\subsection{Deformation by multiple slip}

Until quite recently, dislocation cell structures formed during multiple slip deformation were generally considered to be energetically favourable dislocation arrays with insignificant long-range internal stresses, c.f. [2]. This contrasts the situation in the case of single-slip deformation where long-range internal stresses (due to dislocation pile-ups) had been an important ingredient of stage II work-hardening theories [32,33].

Recent X-ray studies on [001]-orientated copper single crystals deformed in tension at room temperature [4] have provided convincing evidence that significant long-range internal stresses exist also in dislocation cell structures. This conclusion is based on a detailed analysis of the experimental observation that deformation-induced broadening of the $\{002\} X$-ray reflection profiles is asymmetric. For details, the reader is referred to the original paper [4]. Similar results have in the meanwhile also been reported for copper polycrystals deformed in tension at room temperature [34].

These observations which are difficult to interpret in terms of existing dislocation models are easily explained in the composite picture. For this purpose, the model has to be adapted to the geometry of the cell structure and allowance has to be made for multiple slip. Fig. 6 shows an idealized model of the cell structure with two intersecting symmetrically operating slip systems $[1,4]$. Glide dislocations that have traversed the cell interiors are held up at the periphery of the walls (Fig. 6a). In a formal manner, these interface dislocations with Burgers vectors $b_{1}$ and $b_{2}$ can be replaced by others with resultant Burgers vectors b effect of these resultant interface dislocations is to reinforce the applied axial stress $\sigma$ by a forward internal stress $\Delta \sigma_{w}>0$ in the cell walls lying parallel to the stress axis and to reduce $\sigma$ by an internal back stress $\Delta \sigma_{c}<0$ in the cell interiors, as illustrated schematically in Fig. 7. (At the same time the walls lying perpendicular to the stress axis are subjected to internal compressive stresses in the lateral (horizontal) direction.) As in the case of single slip (Sect. 3.1), it must be noted that the number of interface dislocations required to provide a 1 arge enough forward stress $\Delta \sigma_{w}$ in the walls in order to make them transparent to further dislocation glide (macroyielding) is only a small fraction of the total number of dislocations [4]. The insets in Fig. depict schematically the "tetragonal" lattice distortions (referring to an [001]-orientated crystal) retained in the unloaded state in the cell walls lying parallel to the stress axis and in the cell interiors. It is easily shown that the corresponding lattice parameter changes give rise to precisely that $k$ ind of asymmetry of the broadened $X$-ray reflection profiles that was observed $[1,4]$.

With the modifications outlined briefly above, the composite model provides a very satisfactory description of the macroscopic flow stress and the long-range internal stresses in crystals (or crystallites of a polycrystal) deformed in multiple slip at temperatures at which cell structures of the low-temperature type (cf. Fig. 1a) form. Aside from
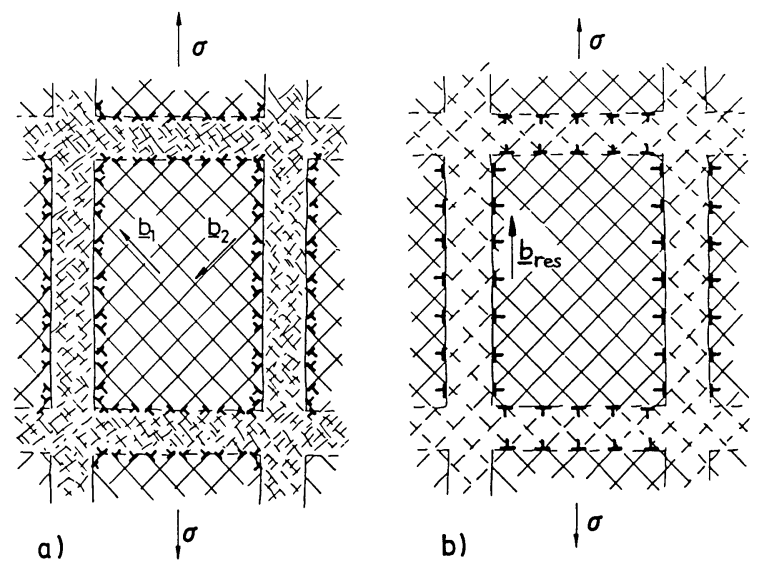

Fig. 6. Model of symmetrical multiple dislocation glide in idealized cell structure. After $[1,4]$.

a) Glide dislocations are held up at the interfaces between cell interiors and walls.

b) Representation of interface dislocations by resultant interface dislocations.

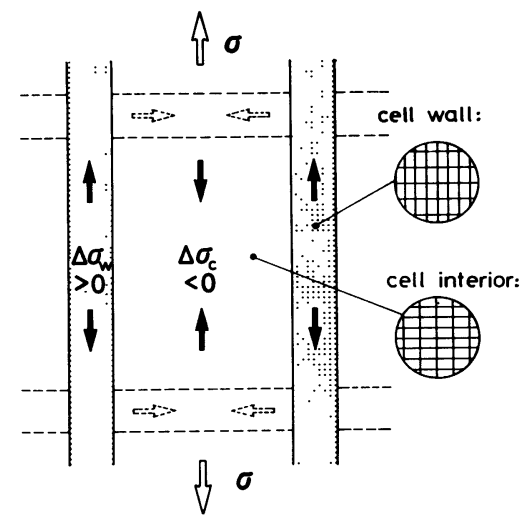

Fig. 7. Schematic illustration of internal stresses in cell walls and in cell interiors with corresponding lattice parameter changes (in the unloaded state). After [4].

details [4], the concept illustrated in Figs. 4 and 5 and the basic equations summarized in Sect. 3.1 are applicable. In this case, the experimental results and theoretical considerations [4] suggest that an Eshelby factor close to one is appropriate. Further work is needed in order to extend the model to dislocation cell structures of the high-temperature type (Fig. Ib).

\subsection{On the sources of the long-range internal stresses.}

As outlined in Sections 3.1 and 3.2 , the message conveyed by the composite model is that, if the dislocation distribution is heterogeneous, then the fulfillment of the compatibility requirements during deformation leads unavoidably to (more or less sig- 
nificant) deformation-induced long-range internal stresses. The importance of this statement lies in the fact that it holds irrespective of whether the bulk of the dislocations, viewed in a "static" sence [1], that is in the absence of the applied stress, are arranged in low-energy configurations or not.

At this stage it is appropriate to identify and classify the dislocation configurations that act as the sources of the long-range internal stresses, as illustrated in Fig. 8 [35]. In a homogeneous dislocation distribution such as the Taylor lattice (Fig. 8a), the range of the elastic strain field is of the order of the mean dislocation spacing and long-range internal stresses are essentially absent. Dislocation pile-ups (Fig. 8b) are a classic representative of a heterogeneous dislocation distribution and, at the same time, the most frequently cited source of long-range internal stresses. However, while dislocation pile-ups certainly play an important role in materials which exhibit planar slip such as $\alpha$-brass or stainless steels, they are a much less common feature in many other materials, and they loose their importance to a large degree in most materials once multiple slip becomes dominant. The dislocation arrangements observed in these latter cases range from(one-dimensional) dislocation bundles and walls to threedimensional cell structures. This class of heterogenous dislocation distribution is represented schematically in Fig. 8c. In this case significant longrange internal stresses arise during deformation, as
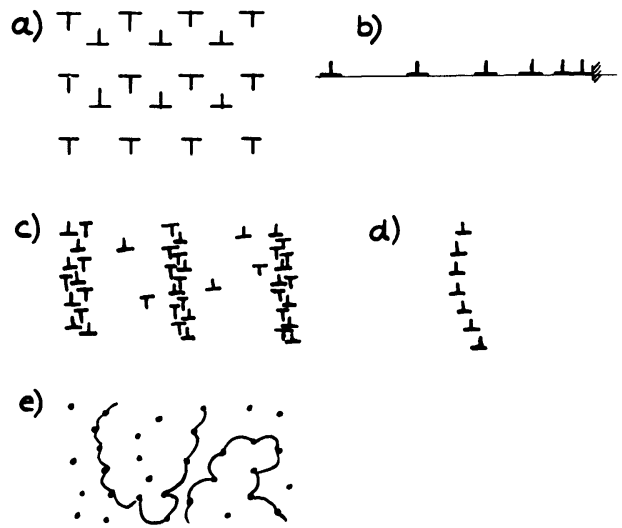

Fig. 8. Schematic survey of dislocation distributions. After [ 35$]$.

a) Regular Taylor lattice.

b) Dislocation pile-up.

c) Dislocation wall (or cell) structure.

d) Flexed dislocation sub-boundary cell wall.

e) Dislocation glide through random obstacle array.

described in Sections 3.1 and 3.2. The sources of these internal stresses have been identified as the so-called interface dislocations $[1,4,19]$. Jackson's recent work [36] (and the work of Pedersen et al. [20], deal in more detail with the specific primary/secondary dislocation distributions such as dislocation sheets (grids) wich are typical of stage II tensile deformation in fcc single crystals. These dislocation configurations fall also into the category of Fig. $8 \mathrm{c}$ and have not been considered here.

In contrast to the heterogeneous dislocation distri- butions discussed so far (Figs. 8b, 8c) which are typical of low-temperature deformation, the dislocation cell walls of the sub-boundary type (Fig. 8d) represent another type of heterogeneous dislocation distribution. Also in this case high local stresses have been detected at or near the walls [37]. The sources of these internal stresses have been ascribed to the flexing of the walls under the action of the applied stress [38] and to dynamic fluctuations in the regularity of the subcell wall network as dislocations are continuously emitted and built in [37]. While these processes are complex, a formal description in terms of a composite model seems possible.

The last case to be considered refers to dislocation glide through a random array of obstacles ( $F i g .8 \mathrm{e}$ ). At first sight, one might expect a quasi-homogeneous dislocation distribution with negligible internal stresses. However, as first pointed out by Orowan [39] in a discussion of the Bauschinger effect, compare Sect. 4, the dislocations sample locally varying inter-obstacle spacings which divide the crystal into soft and hard regions which alternate (with a small wavelength) compare also refs. $[35,40,41]$. Hence a formal similarity to a markediy heterogeneous dislocation distribution exists. The main difference is that in the latter case the hard regions with the small inter-obstacle spacings are localized in the dislocations walls and separated from the soft regions which are localized in the cell interiors, whereas in the case of the random distribution the hard and the soft regions are "intermingled".

Finally, some conclusions seem important. Regardless of the details of the dislocation distribution, internal stresses, defined in a broad sense and with more or less long range, are bound to develop during deformation in all practical cases encountered. Hence, the mechanical behaviour, viewed macroscopically, will be qualitatively similar in most cases. In order to sort out differences with regard to details, the actual dislocation distributions must be studied carefully.

3.4 The effect of the degree of heterogeneity of the dislocation distribution on the flow stress and the elastic energy

In the following we compare in a simple model the properties of a crystal containing a heterogeneous dislocation distribution with that of a reference crystal containing a homogeneous dislocation distribution $[14,35]$. We denote the macroflow stress of the crystal containing a heterogeneous dislocation distribution by $\boldsymbol{\tau}_{\text {tot }}$ which we define according to eq. ( $8 \mathrm{~b})$ and assume that the local flow stresses $\hat{\tau}_{c}$ and $\hat{\tau}_{w}$ are given by relations similar to eq. (4):

$$
\hat{\tau}_{C}=\alpha G b \sqrt{\rho}_{C}
$$

and $\hat{\mathrm{t}}_{w}=\alpha G b \widehat{\rho}_{w}$,

where $\rho_{c}$ and $\rho_{w}$ denote the local dislocation densities in the cell interiors and in the cell walls, respectively. Then the mean dislocation density $p$ can be expressed by a rule of mixtures as

$$
\rho=f_{c} \rho_{c}+f_{w} \rho_{w} \text {. }
$$

The reference flow stress $\hat{\tau}_{\text {hom }}$ of a crystal containing a density $p$ of dislocations which are dis- 
tributed homogeneously is given by the constant value

$$
\hat{\tau}_{\text {hom }}=\alpha G b \sqrt{\rho} \text {. }
$$

With eqs. (8b), (9) and (14) to (17) we obtain

$$
\hat{\tau}_{\text {hom }}^{2}-\hat{\tau}_{\text {het }}^{2}=f_{w} f_{c}(\alpha G b)^{2}\left(r_{\rho_{w}}-\sqrt{\rho}_{c}\right)^{2} \text {. }
$$

Eq. (18) expresses the important fact that, for a given mean dislocation density $\rho$, the flow stress $\tau_{\text {het }}$ for a heterogeneous distribution of the dislocations (for which $P_{w}>P>\rho_{c}$ holds) is always smaller than the flow stress $\hat{\tau}_{\text {hom }}$ for a homogeneous dislocation distribution [14]. Taking the difference between $\rho_{w}$ and $\rho_{c}$ as a measure of the degree of heterogeneity of the dislocation distribution we conclude that, with increasing heterogeneity, $\hat{\tau}_{\text {het }}$ will become increasingly smaller than the constant reference stress $\tau_{\text {hom }}$.

These considerations lead to the macroscopic stressstrain behaviours depicted schematically in Figs. 9a and $9 \mathrm{~b}$ for crystals containing a homogeneous and $a$ heterogeneous dislocation distribution, repectively. The macroscopic flow stresses ' $\hat{\tau}_{\text {hom }}$ and $\hat{\tau}_{\text {het }}<\hat{\tau}_{\text {hom }}$ are compared at a deformation at which the mean dislocation densities are the same in both cases. The correşponding elastic strain energy densities are $\tau_{\text {nom }} / 2 \mathrm{G}$ and $\hat{\tau}_{\text {het }} / 2 \mathrm{G}<\hat{\tau}_{\text {t }} / 2 \mathrm{G}$. These "macroscopic" elastic strain energies are fully recoverable upon unloading, as indicated in Figs. 9a and $9 \mathrm{~b}$.

Viewed microscopically, however, an important distinction must be made. In the case of the heteroge-

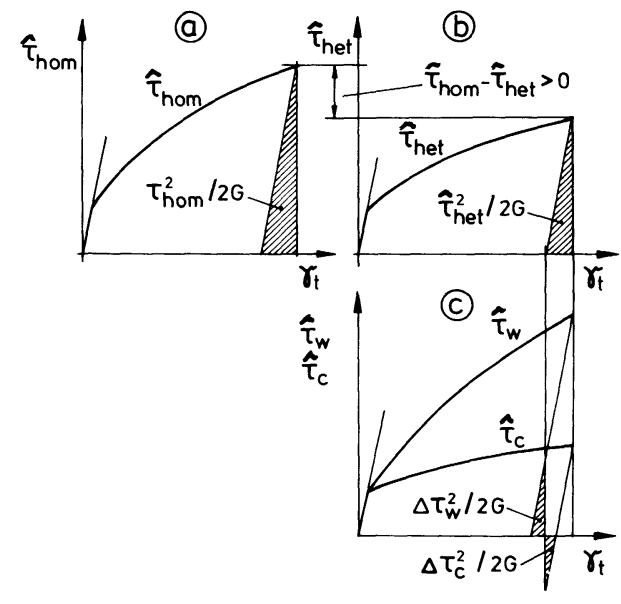

Fig. 9. Stress-strain behaviour and elastic strain energies. After [14].

a) Macroscopic behaviour for homogeneous dislocation distribution.

b) Macroscopic behaviour for heterogeneous dislocation distribution.

c) Microscopic behaviour of walls and cell interiors for heterogeneous dislocation distribution.

neous dislocation distribution, the local microscopic stress-strain behaviours of the cell walls and the cell interiors are as shown in Fig. 9c. The microscopic strain energy density under load is given by $\left(f_{w} \hat{\tau}_{w}+f_{c} \hat{\tau}_{c}^{2}\right) / 2 G$. In addition, it must be noted that, because of the long-range internal stresses, a stored elastic strain energy density $\left(f_{w} \Delta \tau_{w}^{2}+f_{c} \Delta \tau_{c}{ }^{2}\right) / 2 G$ is retained upon unloading. Detailed considerations [14] show that the sum of these two energy terms is independent of the degree of heterogeneity of the dislocation distribution and is in fact constant and equal to

In the simple model discussed, the following conclusion can be drawn. The degree of heterogeneity of the dislocation distribution affects primarily the relative magnitudes of the recoverable and the stored elastic strain energies (the sum remaining constant). With increasing heterogeneity the stored strain energy increases at the expense of the recoverable strain energy $[14,35]$.

A final word is in place regarding the question whether heterogeneous dislocation distributions are low-energy dislocation structures with respect to the stored energy (LEDS [42]) or not. The composite model approach [14] shows that while the elastic strain energy of the dislocation distribution decreases with increasing heterogeneity in the sense of LEDS, the residual elastic strain energy arising from the increasing long-range internal stresses increases in the sense of a high-energy dislocation structure (HEDS). Thus, heterogeneous dislocation distributions must be classified as intermediate-energy dislocation structures (IEDS) in this terminology. At the same time, it must be emphasized that energetic considerations should preferably refer to the stress-applied state which is characteristic of the deformation process [14].

\section{CYCLIC DEFORMATION}

\subsection{The composite model of cyclic plasticity}

The representation of the "hypothetical" stressstrain behaviour of a two-component composite illustrated in Fig. 5 can be extended easily to the case of reverse loading, as shown in $\mathrm{Fig}$. 10. Based on the microscopic behaviour of the components (Fig. 10a), the macroscopic behaviour of the composite (Fig. 1Ob) is obtained under the assumption that no significant microstructural changes occur during forward and reverse straining. This assumption is approximately fulfilled in cyclic saturation. The cyclic hysteresis curve obtained in Fig. 10b exhibits the property of kinematic hardening in association with a Bauschinger effect, i.e. a lowering of the elastic 1 imit after a reversal of the sense of deformation [43]. The dashed "virgin" yielding curve illustrates the yielding of the composite which is considered to be initially free of (long-range) internal stresses. Under the conditions stated, the cyclic hysteresis curve is related uniquely to the virgin monotonic yielding curve and can be obtained from the latter by multiplication of the stresses and the strains by a factor of two. Materials obeying this rule are said to exhibit Masing behaviour [28].

Fig. 10b refers to the case where the (plastic) strain amplitude is so large that the hard component yields plastically. In general, two cases must be considered in the case of a two-component composite, as illustrated in Figs. $11 \mathrm{a}$ and $11 \mathrm{~b}[1,19]$. When $\varepsilon<\hat{\sigma}_{1} / E$, where $E$ is Young's modulus, a slim sharply pointed hysteresis "loop", characteristic of cyclic microplasticity is obtained (Fig. 11a), whereas for larger amplitudes $\left(\varepsilon_{t}>\hat{\sigma}_{1} / E\right)$ the hysteresis loop assumes the "more rounded" shape characteristic of cyclic macroplasticity (Fig. 11b). 


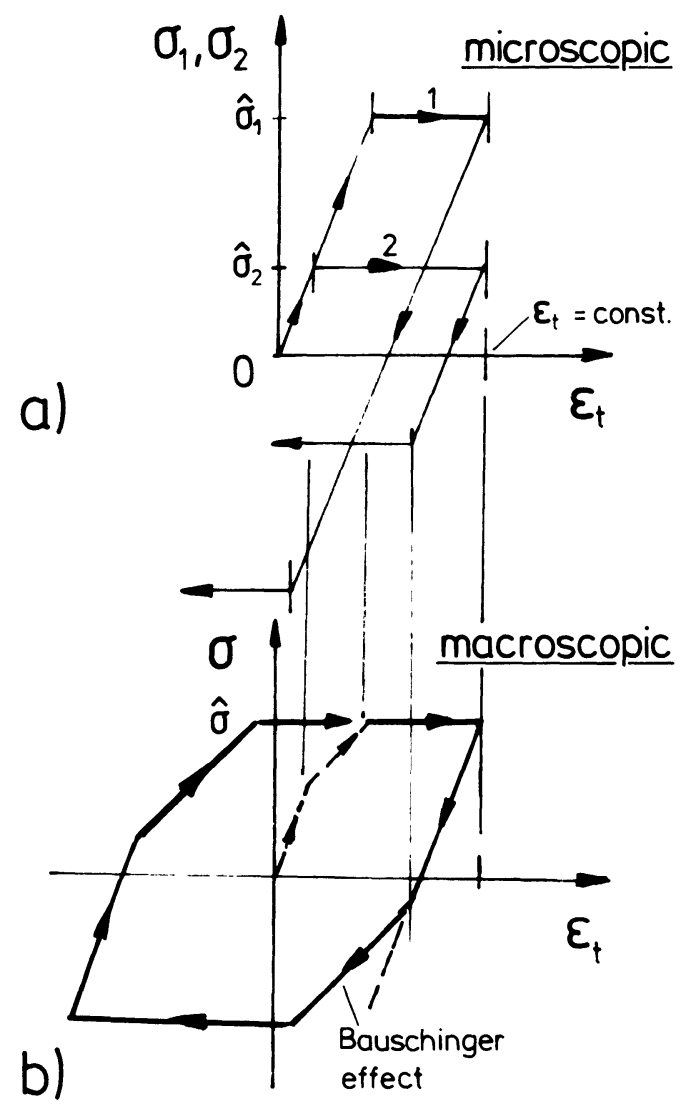

Fig. 10. Cyclic stress-strain behaviour of a twocomponent composite. After [19].

a) Microscopic behaviour of the components.

b) Macroscopic behaviour of the composite.

\subsection{Permanent softening}

In the case of cyclic microplasticity, as defined above, the hysteresis loop exhibits the phenomenon of so-called permanent softening. When the compressive deformation is plotted in the first quadrant by plotting the magnitude of the stresses against the cummulative strain, the stress in compression lies below the extrapolated branch of the tensile deformation by a constant amount $\Delta \sigma_{p}$. A more detailed consideration shows that, expressed as a shear stress $\Delta \tau_{p}$ as in eq. (11), the permanent softening is:

$$
\Delta \tau_{p}=2\left|\Delta \tau_{2}\right|=2 \Gamma \mathrm{rf}_{1}\left(\gamma_{\mathrm{pl}, 2}-\gamma_{\mathrm{pl}, 1}\right) \text {. }
$$

Here $\Delta \tau_{2}$ is the long-range internal back stress in the soft components 2, frequently also referred to as the mean stress $[20,30,41]$. This relationship has proved very valuable in the work of Brown and Stobbs [30] on dispersion-hardened copper single crystals which exhibit a very marked permanent softening and where the permanent softening $\Delta T_{\text {p }}$ has been found to be related intimately to the Pongrange internal stresses. On the other hand, the absence of permanent softening in the case of single-phase materials does not justify the conclusion that long-range internal stresses are insignificant. Rather, the situation then can be similar to

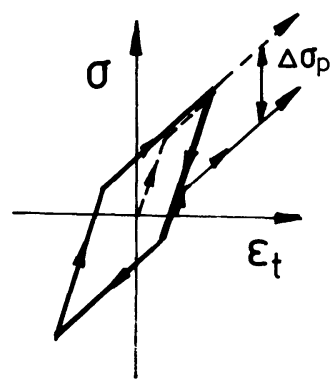

a)

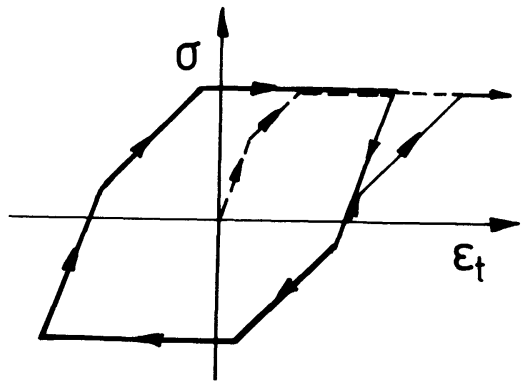

b)

Fig. 11. Cyclic hysteresis "loops" characteristic of cyclic micro- and cyclic macroplasticity.

a) Cyclic microplasticity with permanent softening.

b) Cyclic macroplasticity without permanent softening.

that shown in Figs. 10 and $11 \mathrm{~b}$, and in this case of cyclic macroplasticity, the iong-range internal stresses are very significant indeed and in fact so large that the hard components 1 , i.e. the dislocation walls, yield under the combined action of the applied and the forward internal stress $\Delta \tau_{1}$, compare eqs. (12) and (13).

\subsection{Application of the composite model to the cyclic plasticity of fatigued copper single crystals}

Several authors have analyzed the cyclic plasticity of fatigued metals in terms of different types of composite models $[18,19,21-23]$. The present author has applied the simple concepts outlined in Sections 4.1 and 4.2 in order to model the cyclic plasticity of the matrix and of the PSB wall structures in copper single crystals fatigued into cyclic saturation $[1,18,19]$. The hysteresis loop of the matrix was found to be of the type illustrated in Fig. 11a, suggesting that in the range of cyclic microplasticity the dipolar veins act as hard undeformable regions. On the other hand, the hysteresis loop of the PSB wall structure was concluded to be approximated well by a loop characteristic of cyclic macroplasticity as in Fig. 11b, implying that the walls yield plastically. The hysteresis loops obtained in these studies were necessarily polygons characteristic of the simple two-component composite model used. In the following the model will be extended in order to analyze a particularly simple case of a hysteresis curve of a fatigued copper crystal.

The example chosen refers to the hysteresis curve of a copper single crystal fatigued into cyclic saturation at room temperature at a plastic shear strain amplitude $\gamma_{\mathrm{pl}}=7.5 \cdot 10^{-3}$ with a saturation shear 
stress amplitude $\tau_{s}=29.8 \mathrm{MPa}$ [44]. At this particular strain amplitude the dislocation microstructure is completely deprived of the matrix and consists exclusively of the PSB wall structure $[18,19,24,44]$. Fig. 12 shows the hysteresis loop in a plot of shear stress $T$ versus plastic shear strain $\gamma$. It is clear that in order to model such a smoothly curved hysteresis loop satisfactorily, a composite model with more than two components is required. We therefore allow for a spectrum of local flow stresses $\hat{\tau}_{w}$ in the walls and $\hat{C}$ in the channels, respectively. In the present case, this approach is considered to be adapted better to the basically two-component nature of the dislocation wall structure than the use of a probability density function as in related models of (polycrystal) cyclic plasticity $[22,23]$.

We assume that the hysteresis loop obeys Masing behaviour. Then the shear stress, measured from the tip of the loop, is equal to $2 \tau$. We denote the volume fraction of material that is deforming plastically at a particular stress level by $f_{p l}=f_{p l}(2 \pi)$. It is then easy to show that (for $\Gamma=1$ ) in a plot of $\tau$ versus $\gamma_{p l}$ as in Fig. 12, the slope at a particular value of $f_{p l}$ (or $\tau$ ) is given by

$$
\frac{d_{\tau}}{d_{\gamma_{p l}}}=\frac{1-f_{p l}}{f_{p l}} \cdot G .
$$

Hence

$$
f_{p 1}=\frac{1}{\frac{1}{G} \cdot \frac{d \tau}{d \gamma_{p 1}}+1} .
$$

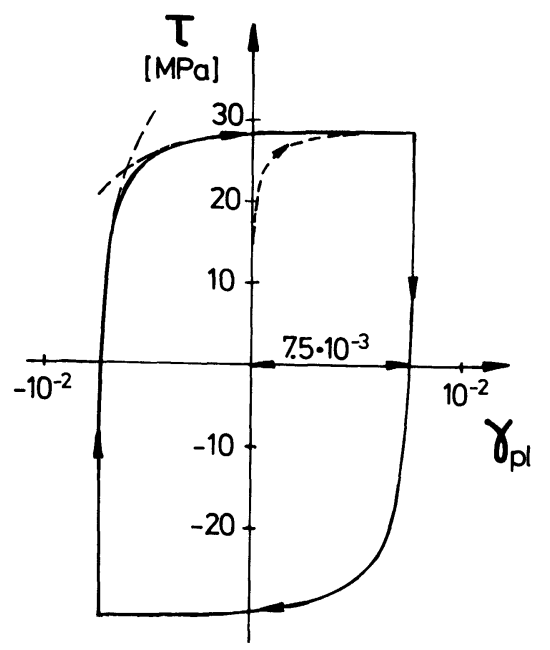

Fig. 12. Hysteresis curve of copper single crystal fatigued at $\gamma_{\mathrm{pl}}=7.5 \cdot 10^{-3}$ into cyclic saturation $[18,44]$.

Thus, if $f_{p l}(2 \tau)$ is known, the hysteresis loop is obtained by integration of eq. (20). On the other hand, for any given loop, $f_{p l}$ can be determined readily by a graphical method according to eq. (21). In our case, $f_{p l}$ is composed of two contributions, namely $f_{p l, c}$ from the channels and $f_{p l, w}$ from the walls:

$$
f_{p l}=f_{p l, c}+f_{p l, w} \text {. }
$$

Fig. 13 illustrates the model. As the stress is increased (in tension or in compression), measured from the tips of the hysteresis 10op, $f_{p}$ and its components $f_{p l}, c$ and $f_{p}$, wherease as function
of $2 \tau$ as indicated. Pf the functions $f$ and $f_{p l, w}$ are separated sufficiently along the stress axis, then the resultant curve $f_{p l}$ should exhibit two points of inflection. Note that, in the simple two-component composite model (Sections 4.1,4.2), the functions $f_{p l}, c$ and $f_{p l, w}$ would be stepfunctions with a jump from zero to $f_{c}$ and $f_{w}$ at the discrete stress values $2 \tau_{c}$ and $2 \hat{\tau}$, respectively.

The result of the evaluation of the hysteresis loop shown in Fig. 12 along these lines is shown in Fig. 14. The rather big scatter of the data points in the range $2 \tau \leqslant 35 \mathrm{MPa}$ is due to the inaccuracy of the evaluation of the very steep slope in this stress range. Nonetheless, the curve drawn through the data points exhibits clearly the features expected according to the model with one inflection point at $2 \tau \approx 35 \mathrm{MPa}$ and another one at $2 \tau \approx 48 \mathrm{MPa}$. In our model, the second inflection point denotes roughly that external stress level at which the weakest walls begin to yield plastically. Hence the fact that such an inflection point exists provides further evidence that the walls yield plastically and that the Eshelby factor $T$ must be close to one, compare Section 3.1. One might expect naively that the second inflection point can be anticipated by more inspection of the hysteresis loop. However, the hysteresis loop displays no discontinuity except at the tips. At best, it can be stated that the stress level at which the second inflection point appears is close to the point of intersection of the extrapolated curves that approximate the ascending and the almost horizontal parts of the curve, cf. Fig. 12 .

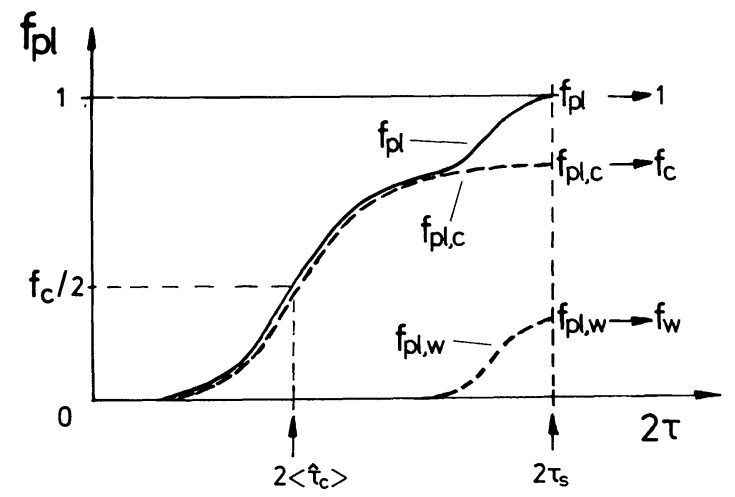

Fig. 13. Model showing how the volume fraction $f_{p l}$ of plastically deforming material increases as $\mathrm{pl}$ function of increasing stress level.

The detailed evaluation is performed as follows. The curve extending up to $2 \tau \approx 45 \mathrm{MPa}$ is considered to represent essentially only the function $f_{p l}, c^{\circ}$. It is assumed that the value of $f_{p l}$ obtained by the extrapolation of this curve up to the saturation stress level $2 \tau_{s}$ is identical with $f_{c}$. A value of $f_{c}=0.87$ is obtained. The function ${ }^{c} f_{\text {s }}$ is found by subtracting the extrapolated curve f $f$ from $f_{p l}$. We note in passing that the shape of the curve $f^{p} 1$ correlates rather well with the curve obtained from the distribution of the bowing stresses (necessary to drive the screw segments along the channels) based on the variations of the channel 
widths $d_{c}$ [19]. Next, a mean value $2\left\langle\hat{\tau}_{c}\right\rangle$ is defined somewhat arbitrarily for $f_{p l}=f_{c} / 2$. This procedure yields $\left\langle\hat{\tau}_{\mathrm{c}}\right\rangle=18 \mathrm{MPa}$. In order to obtain the corresponding mean value $\left\langle\hat{\tau}_{w}\right\rangle$, eq. (8b) is solved at the saturation stress level ${ }^{\prime} \tau_{s}=\tau$ for $\left\langle\hat{\xi}_{w}\right\rangle$, using the previgusly found values $f_{c}$, $\left\langle\tau_{W}\right\rangle \approx 109 \mathrm{MPa}$. It should be noted that, in the present evaluation, $\left\langle\hat{\tau}_{r}\right\rangle$ and $\left\langle\hat{\tau}_{W}\right\rangle$ consist not

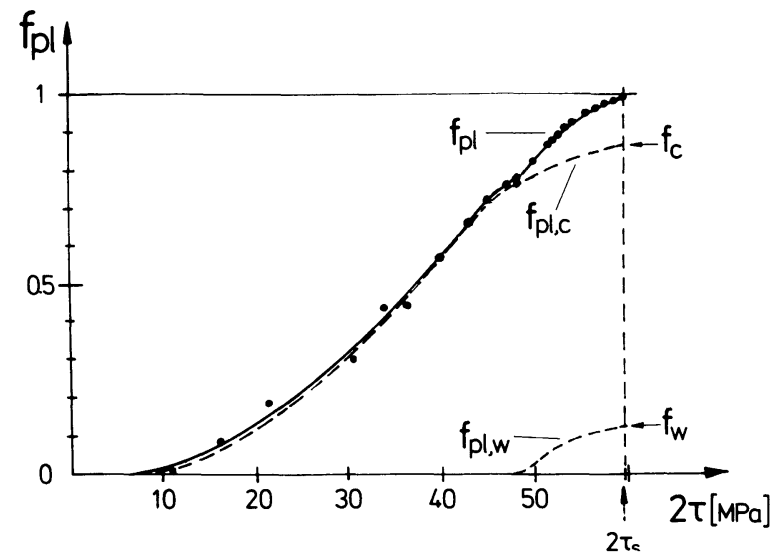

Fig. 14. Evaluation of the hysteresis curve shown in Fig. 12 according to the model illustrated in Fig. 13. A value of $G=42000 \mathrm{MPa}$ was used.

only of the dominant athermal component but contain also a (much smaller) thermal component.

The hysteresis curve in Fig. 12 is now well characterized by $f_{f}=0.87(0.9), f_{w}=0.13(0.1)$, $\left\langle\hat{\tau}_{c}\right\rangle=18 \mathrm{MPa}(20 . f \mathrm{MPa})$ and $\left\langle\hat{\tau}_{W}^{W}\right\rangle=109 \mathrm{MPa}$ (113 $\mathrm{MPa})$. The values in brackets are listed for comparison. They were obtained previously by combining the results of TEM observations with the simple two-component composite model and by making use only of the saturation stress value [19]but not of the actual hysteresis curve shape as was done here. The good agreement between the results of these two independent approaches is very gratifying. It is clear then that the next step should be to extend the present monocrystal model to polycrystals.

\section{CONCLUDING REMARKS}

A description has been given of the monotonic and cyclic deformation behaviour of crystals containing heterogeneous distributions of dislocations. It has been shown that the composite model approach possesses distinct advantages compared to models which attempt to consider a number of individual dislocation mechanisms. In particular, the straight-forward interpretation of long-range internal stresses and the applicability to reverse loading are noteworthy. As they stand, the composite models provide good descriptions as a first order approximation. With further refinements, it should be possible to widen considerably the scope of applicability of the composite models.

\section{Acknowledgment}

The author expresses his gratitude to Frau G. Raffel, Frau W. Teichman and Frau A. Weiss for their help in preparing the camera-ready manuscript and the figures.

\section{REFERENCES}

[1] H. Mughrabi, Acta metall. 31 (1983) 1367 and unpublished work.

[ 2] N. Hansen and D. Kuhlmann-Wilsdorf, in Proc. of Int. Conf. on Low Energy Dislocation Structures, Charlottesville, Va, Aug. $10-14$, 1986, Mat. Sci. Eng. 81 (1986) 141.

[3] C. Laird, P. Charsley and H. Mughrabi, ibid., Mat. Sci. Eng. 81 (1986) 433

[a] H. Mughrabi, T. Ungár, W. Kienle and M. Wi1kens, Phil. Mag. A, 53 (1986) 793.

[5] G. König and W. Bium, Acta metall 28 (1980) 519.

6] O.K. Chopra and C.V. Gowda, Phil. Mag. 30 (1974) 583.

7] H. Mughrabi, K. Herz and X. Stark, Int. J. Fract. 17 (1981) 193.

[8] R. Wang and H. Mughrabi, Mat. Sci. Eng. 63 (1984) 147.

[ 9] E. Göttler, Phil. Mag. 28 (1973) 1057.

[10] U. Essmann and H. Mughrabi, Phil Mag. A, 40 (1979) 731.

[11] F.R.N. Nabarro, Z.S. Basinski and D.L. Holt, Adv. in Phys. 13 (1964) 193.

12] G. Schoeck and R. Frydman, phys. stat. sol. (b), 53 (1972) 661 .

13] W. Púsch1, R. Frydman and G. Schoeck, phys. stat. sol. (a), 74 (1982) 211.

14 ] H. Mughrabi, Mat. Sci. Eng. 85 (1987) 15.
[15] H. Mughrabi and R. Wang, in Proc. of First Int. Symp. on Defects and Fracture, Tuczno, Pol and, Oct. 1980, edited by G.C. Sih and H. Zorski, Martinus Nijhoff Publ., 1982, p. 15.

[16] F.B. Prinz and A.S. Argon, Acta metall. 32 (1984) 1021.

[17] W.D. Nix, J.C. Gibeling and D.A. Hughes, Met. Trans. 16A (1985) 2215.

[18] H. Mughrabi, in Proc. of $5^{\text {th }}$ Int. Conf. on the Strength of Metals and Alloys, Aachen, 1979, edited by P. Haasen, V. Gerold and G. Kostorz, Vol. 3, Pergamon Press, 1980, p. 1615.

[19] H. Mughrabi, in Continuum Models of Discrete Systems 4 , edited by 0 . Brul in and R.K.T. Hsieh, North-Holland Publishing Company, 1981, p. 241, and unpublished work.

[20] O.B. Pedersen, L.M. Brown and W.M. Stobbs, Acta metall. 29 (1981) 1843.

[21] O.B. Pedersen and A.T. Winter, Acta metal1. 30 (1982) 711

[22] C. Holste and H.-J. Burmeister, phys. stat. sol. (a) 57 (1980) 269 .

[23] J. Polák and M. Klesnil, Fat. Eng. Mat. Struct. 5 (1982) 19.

[24] H. Mughrabi, K. Herz and F. Ackermann, in Fatigue Mechanisms, edited by J.T. Fong, ASTM STP 675 , p. 69.

[25] P.J. Woods, Phil. Mag. 28 (1973) 155. 
[26] J.C. Grosskreutz and H. Mughrabi, in Constitutive Equations in Plasticity, edited by A.S. Argon, MIT Press, Cambridge, Mass., 1975, p. 251.

[27] H. Mughrabi, in Proc. of $3^{\text {rd }}$ Int. Conf. on the Strength of Metals and Alloys, Cambridge, Vol. 1. 1973 , p. 407.

[28] G. Masing, Wissenschaftl. Veröffentl. aus dem Siemens-Konzern 3 (1923) 231.

[29] J. D. Eshelby, P Froc. Roy. Soc. London (A), 241 (1957) 376.

[30] L.M. Brown and W.M. Stobbs, Phil. Mag. 23 (1971) 1185.

[31] J. Lepinoux and L.P. Kubin, Phil. Mag. A, 51 (1985) 675 .

[32] A. Seeger, J. Diehl, S. Mader and H. Rebstock, Phil. Mag. 2 (1957) 323.

[33] P.B. Hirsch and T.E. Mitchell, Can. J. Phys. 45 (1967) 663.

[34] T.ungár, S. Tóth, J. I1ly and I. Kovács, Acta meta11. 34 (1986) 1257.
[35] H. Mughrabi, S. Afr. J. Phys. 9 (1986) 62.

[36] P.J. Jackson, Acta metall. 33 (1986) 449.

[37] M.A. Morris and J.L. Martin, Acta metail. 32 (1984) 549.

[38] A.S. Argon and S. Takeuchi, Acta metal1. 29 (1981) 1877 .

[39] E. Orowan, in Internal Stresses and Fatigue of Metals, edited by G.M. Rassweiler and W.L. Grube, El sevier, Amsterdam, 1959, p. 59.

[40] U.F. Kocks, Phil. Mag. 13 (1966) 541

[41] L.M. Brown, Scripta metal1. 11 (1977) 127.

[42] Low Energy Dislocation Structures, Proc. of Int. Conf., Charlottesville, Aug. 10-14, 1986, edited by M.N. Bassim, W.A. Jesser, D. Kuhlmann-Wilsdorf and H.G.F. Wilsdorf, Mat. Sci. Eng. 81 (1986).

[43] J. Bauschinger, Mittheilungen aus dem Mechanisch-Technischen Laboratorium der K. Techn. Hochschule München 13 (1886) 1.

[44] H. Mughrabi, Mat. Sci. Eng. $33^{\circ}$ (1978) 203. 